\title{
Sirolimus treatment for cirrhosis or hepatocellular carcinoma patients accompanied by psoriasis after liver transplantation: A single center experience
}

\author{
LIN ZHOU $^{1,2^{*}}$, GUO-SHENG DU ${ }^{2 *}$, LI-CHAO PAN ${ }^{1 *}$, YONG-GEN ZHENG ${ }^{1,2}$, ZHI-JIA LIU ${ }^{2}$, HAI-DA SHI ${ }^{1}$, \\ SHAO-ZHEN YANG ${ }^{2}$, XIAN-JIE SHI ${ }^{1}$, MENG XUAN ${ }^{1}$, LI-KUI FENG ${ }^{2}$ and ZHI-DONG ZHU ${ }^{2}$ \\ ${ }^{1}$ Department of Hepatobiliary Surgery, Chinese PLA General Hospital, Beijing 100853; ${ }^{2}$ Department of Hepatobiliary Surgery, \\ Organ Transplant Institute, Chinese PLA 309th Hospital, Beijing 100091, P.R. China
}

Received February 28, 2017; Accepted October 6, 2017

DOI: $10.3892 / \mathrm{ol} .2017 .7217$

\begin{abstract}
There is currently no consensus on the most suitable therapeutic approach for psoriasis (PS) co-existing with posthepatic cirrhosis (PCs) and hepatocellular carcinoma (HCC) following liver transplantation (LT). The present study provides an analysis of the therapeutic experience of such patients. Five LT recipients (two with PC and three with HCC) with accompanying PS were included. The induction program consisted of methylprednisolone plus basiliximab treatment. The initial postoperative treatment scheme consisted of tacrolimus (FK506) plus mycophenolate
\end{abstract}

Correspondence to: Professor Guo-Sheng Du, Department of Hepatobiliary Surgery, Organ Transplant Institute, Chinese PLA 309th Hospital, A-17 Heishanhu Road, Haidian, Beijing 100091, P.R. China

E-mail: duguosheng@medmail.com.cn

Professor Xian-Jie Shi, Department of Hepatobiliary Surgery, Chinese PLA General Hospital, 28th Fuxin Road, Haidian, Beijing 100853, P.R. China

E-mail: shixianjie301@126.com

*Contributed equally

Abbreviations: AAD, American Academy of Dermatology; AID autoimmune disease; CsA, cyclosporine; CT, computed tomography; FK506, tacrolimus; HBIG, human hepatitis B immunoglobulin; $\mathrm{HBV}$, hepatitis B virus; $\mathrm{HCC}$, hepatocellular carcinoma; HCV, hepatitis $\mathrm{C}$ virus; IFN- $\alpha$, interferon $\alpha$; IL, interleukin; LT, liver transplantation; MMF, mycophenolate mofetil; MRI, magnetic resonance imaging; mTOR, mechanistic target of rapamycin; PASI, psoriatic area and severity index; PBC, primary biliary cirrhosis; PCs, posthepatic cirrhosis; PS, psoriasis; SRL, sirolimus; TNF- $\alpha$, tumor necrosis factor $\alpha$; ALD, autoimmune liver disease; ALT, alanine aminotransferase; DBIL, direct bilirubin; TBIL, total bilirubin; $\gamma$-GGT, $\gamma$-gltamyltranspeptidase; ALP, alkaline phosphatase; AFP, alpha fetoprotein; IS, immunosuppressant.

Key words: sirolimus, psoriasis treatment, tacrolimus, liver transplantation, immunosuppression mofetil (MMF) and hormone; the latter was withdrawn 1 week after LT. The patients with PC had been using FK506 with or without a postoperative MMF program; the patients with HCC and recurrence of PS had been switched to a sirolimus (SRL)-based replacement therapy. Furthermore, all patients received anti-hepatitis $B$ virus (HBV) therapy. The patients were followed up after $8.3 \pm 1.5$ years. There was a positive correlation between HBV-DNA copy numbers, and psoriatic area and severity index (PASI) scores $(\mathrm{r}=0.97 ; \mathrm{P}=0.006)$. The PASI scores were decreased significantly at 6 months following surgery compared with pre-transplantation $(\mathrm{P}<0.05)$. The patients who had received the FK506-based treatment experienced PS recurrence two years post-transplantation. The PASI scores increased significantly $(\mathrm{P}<0.05)$ and then declined gradually, maintaining a stable level $(\mathrm{P}<0.05)$ by 1 year after switching to the SRL-based treatment. The patients who had received the SRL-based treatment exhibited no recurrence of PS. The results of the present study suggest that SRL therapy provides a promising novel treatment method for patients with PS following LT that may be superior to tacrolimus treatment. When co-existing HBV is present pre-transplantation, regular injection of human hepatitis B immunoglobulin should be used to prevent the HBV from relapsing or aggravating the PS.

\section{Introduction}

Psoriasis (PS) is one of the most frequent dermatological diseases, shows polygenic inheritance, and occurs in approximately $2 \%$ of the population $(1,2)$. The etiology and pathogenesis is not yet clear, and may be associated with virulence genes, environmental factors and immunological derangement. Recently, increasing evidence suggests that changes of skin microbiota, especially Firmicutes, may contribute to the pathogenesis of PS. However, it is not known whether these alterations in the microbiome are a consequence of PS itself. Nevertheless, consideration of the microbiome provides a new outlook on PS; the microbiota may be associated with the inflammatory reaction and therefore should be considered in the treatment of PS (3).

PS is believed to be an autoimmune disease (AID) with an inherited genetic predisposition, and is potentially mediated 
by multiple immune cells, such as dendritic cells, T cells and spongiocytes, as well as pro-inflammatory cytokines produced by these cells $(4,5) . \mathrm{CD}^{+} \mathrm{T}$ cells, which represent the autoimmune core of the disease, drive the initial phase of PS and act as a repository of specific disease memory $(4,6)$. Several recent studies have demonstrated that interleukin (IL)-17-producing $\mathrm{CD}^{+}$and $\mathrm{CD}^{+} \mathrm{T}$ cells may act as critical factors in driving the autoimmune reaction during both the initial phase of PS and the subsequent proinflammatory loop (6). Furthermore, it has been suggested that other cytokines such as IL-23/9/22, tumor necrosis factor $\alpha(\mathrm{TNF}-\alpha)$ and interferon $\alpha(\mathrm{IFN}-\alpha)$ may also play important roles in the pathogenesis of PS (6-8). Recently, more attention has been given to the IL-23/Th17 axis, which is believed mainly to play an important role in controlling the proinflammatory loop in psoriatic plaques (7). TNF- $\alpha$ and IFN- $\alpha$ have been recognized as exacerbation factors in PS progression, and play important roles in the inflammatory cascade that leads to different symptoms, ranging from skin lesions to arthritis $(8,9)$. The concept of $\mathrm{T}$ cell involvement in the pathogenesis of PS is supported by the beneficial effect of using cyclosporine (CsA) and tacrolimus (FK506), both of which suppress $\mathrm{T}$ cell activation (10). Recently, it has been demonstrated that adalimumab therapy is also beneficial in the control of PS (11). It acts by suppressing T cells and decreasing plasma IL-17 levels and IL-17-positive cells in psoriatic lesional skin (11).

Infection is believed to be a key risk factor for inducing or aggravating the disease (12). In addition, the younger the patient, the more serious the illness is, and the more prone the patient is to relapse (13). Provoking infections have been reported to appear in more than $40 \%$ of PS patients (14). There is increasing evidence indicating that both bacterial and viral infection could play important roles in the pathophysiology of PS $(15,16)$. In addition, as PS has an inherited genetic predisposition, immunological derangement, AID status and other factors may also play a crucial role in its pathogenesis. The co-existence of PS with AID, primary biliary cirrhosis (PBC), primary sclerosing cholangitis, and autoimmune hepatitis have been reported frequently (17-19). A total of $13 \%$ of patients with PBC have co-existing PS, which is more than 6 times greater than the incidence in the general population (18).

PS is a type of immune inflammatory disease that involves multiple factors, which contributes to the lack of effective drugs and therapeutic approaches (20). Although there are literature reports that long-term application of an immunosuppressant after liver transplantation (LT) is helpful in the treatment of PS and can restrain disease progress and relapse to a certain extent, there are still patients that undergo PS recurrence, progression, and delayed healing after LT, which seriously affect the liver graft's and patient's survival. The evidence associated with the treatment for co-existing PS after LT is scarce and mostly consists of case reports $(21,22)$. Furthermore, if the primary disease is preoperatively differentiated, there is no clear guidance on the selection of postoperative immunosuppression treatment. Therefore, the aim of this study was to analyze the therapeutic experience of LT patients with different pathologies [posthepatic cirrhosis (PCs) or hepatocellular carcinoma (HCC)] co-occurring with PS, thereby increasing the evidential support for different treatment options.

\section{Materials and methods}

Basic data. Five LT patients with accompanying PS were selected for this study from a total of 617 LT patients. All 5 patients were preoperatively positive for hepatitis $B$ virus (HBV). Their average age was $51 \pm 3$ years (male:female, $3: 2$ ). In terms of primary disease, two were diagnosed with PCs and the other three had hepatitis B cirrhosis complicated with HCC, with no accompanying autoimmune liver disease (ALD). All 5 patients were diagnosed with PS before LT, and no de novo PS after LT was reported. This study approved by the Ethics Committee of Human Experimentation of the PLA 309th Hospital (Beijing, China). Written informed consent was obtained in accordance with the Declaration of Helsinki of the World Medical Association.

Patients' history and physical findings. All 5 patients had a 6-10 years PS history (mean, $8.10 \pm 1.52$ years) and a long chronic hepatitis history beyond 10 years (median, 24.80 \pm 2.86 years). All were preoperatively confirmed to be HBV-positive (Table I). Anti-HBV therapy included lamivudine or entecavir when the patients exhibited PCs.

The PS lesions were multiple, bright-colored, red alternating with white, diffuse and flushing. They gradually emerged from the head and face to the neck and trunk with no obvious inducement. Other lesion features were furfuraceous scales, and obsolete scales attached to the surface of lesions, with different degrees of desquamation, not involving the mucosa and submucosa. The PS lesions in all patients were mainly distributed on the trunk, and without regular treatment, there were no cured, old, or new lesion alternations caused by the long, protracted course of the disease.

The mean psoriatic area and severity index (PASI) score was $41.4 \pm 6.9$ (PASI scores are the most commonly used clinical measure of PS, with values ranging from 0 to 72 ) (Table I). Patients with PCs presented liver function and blood coagulation dysfunction along with ascites and malnutrition or hypoproteinemia. Moreover, patients with PCs and HCC also showed high levels of $\alpha$ fetoprotein and hepatic mass signals in abdominal computed tomography (CT) and magnetic resonance imaging (MRI) images.

As the confirmation of a PS diagnosis is usually based on clinical features, including skin rashes, predilection sites and seasonal onset, most diagnosis is not difficult. The cultivation of the dander which performed by the methods of real-time quantitative PCR and immunohistochemistry usually used to detect the expression of HBV-DNA or other viruses $(16,23-25)$ and the expression trends of IL-17/23 $(7,11)$ or TNF- $\alpha$ and INF- $\gamma$ (6-8). So, when these patients were treated in our center, they all had a lengthy PS history and had been treated in hospital, our diagnosis was based on the earlier diagnosis and treatment of patients by a specialist. Meanwhile, combined with the patient's history including the PASI score, we confirmed the diagnosis and excluded other nonspecific infections with the laboratory (hematological examination, cultivation of the dander $(11,23,24,26,27)$ and imaging (CT and MRI scanning) diagnosis methodology.

Operation and therapeutic approaches. The 5 selected patients were well-prepared for LT. Methylprednisolone 
plus basiliximab was used as the preoperative induction treatment. FK506 plus mycophenolate mofetil (MMF) and hormone was used as the initial scheme in the early-stage postoperative period, with hormone withdrawal 1 week after LT (Table I).

The classification and selection standard of the therapeutic approach was based on current, internationally-recognized treatment strategies for patients with HCC or not, when performed following LT (the HCC patients accepted sirolimus (SRL)-based immunosuppressive therapy after LT, and the non-HCC patients were treated with tacrolimus-based immunosuppressive therapy). Therefore, the 2 patients with HBV and PCs had been using FK506 with or without a postoperative MMF program. The others patients, with HBV cirrhosis and HCC, had been switched to a SRL-based replacement therapy within 1 month after LT. Moreover, FK506 was gradually reduced and withdrawn at six months after LT (Table I).

In addition, all 5 patients were given anti-HBV therapy to prevent hepatitis $B$ recurrence, via intravenous injection of human hepatitis B immunoglobulin (HBIG) to maintain effective blood antibody titers.

Index analysis. Basic data including liver function, rejection, and survival time were analyzed in LT recipients with PS. PASI scores for the 5 cases were compared before and after LT. Meanwhile, the adjustment of immunosuppressant was summarized for different patients with PS.

Statistical analysis. The data were analyzed with SPSS 19.0 software (IBM SPSS, Armonk, NY, USA). The measurement data are shown as mean \pm standard deviation, and were compared with Student's t-tests; in addition, count data were compared with Chi-square tests. Differences with $\mathrm{P}<0.05$ were considered statistically significant.

\section{Results}

Basic information. All patients were regularly followed up postoperatively, and had survival periods of $8.3 \pm 1.5$ years. There were no obvious acute rejections during the follow-up time, nor were there signs of hepatitis B or tumor recurrence. The general information and pathological findings are shown in Table I. The main liver function indexes, alanine aminotransferase (ALT), direct bilirubin (DBIL), total bilirubin (TBIL), $\gamma$-gltamyltranspeptidase $(\gamma$-GGT), and alkaline phosphatase (ALP), were recorded for 1 month (Table II; Fig. 1).

PASI changes. Compared with their preoperative condition, the PASI scores of patients reduced significantly during the first 6 months after LT $(\mathrm{P}<0.05)$. However, the two HBV and PCs patients underwent PS recurrence after 2 years, resulting in PASI score increases. After switching to an SRL-based treatment, the PASI scores were gradually decreased and maintained at a steady level from the third year onwards, with no increases to date. The PASI score changes of the 5patients before and after LT are shown in Fig. 2. Furthermore, the preoperative HBV-DNA copy numbers were $(6.9 \pm 3.2) \times 10^{7}$, and showed a positive correlation with the PASI scores $(r=0.97$, $\mathrm{P}=0.006$ ) (Fig.3). 


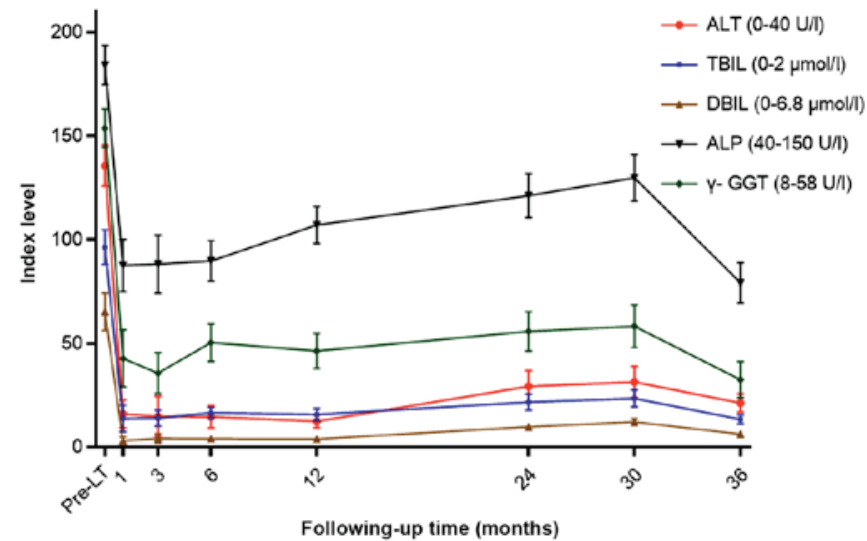

Figure 1. Liver function changes in the recipients of liver transplantation with psoriasis, at different time points. LT, liver transplantation; ALT, alanine aminotransferase; TBIL, total bilirubin; DBIL, direct bilirubin; ALP, alkaline phosphatase; $\gamma$-GGT, $\gamma$-gltamyltranspeptidase.

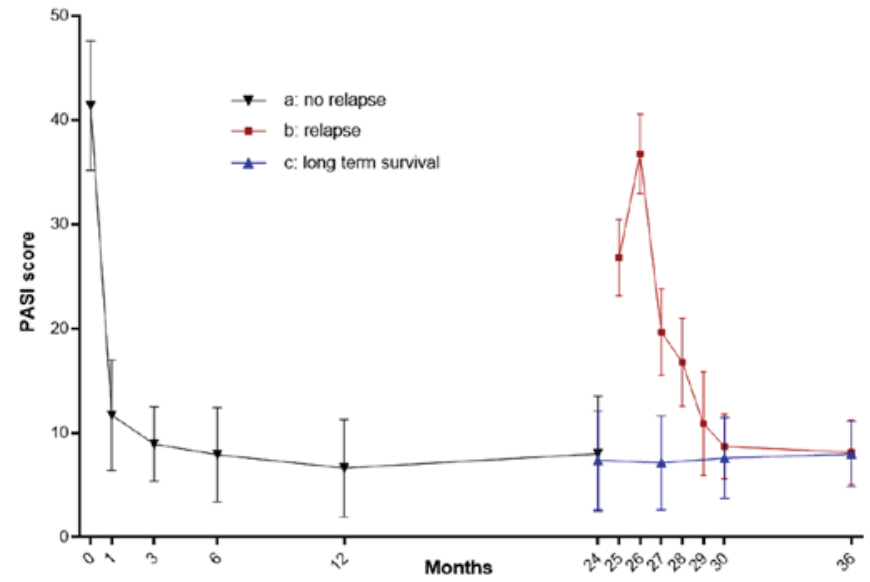

Figure 2. Changes of the PASI score for five recipients after liver transplantation. a, the mean PASI score of five patients showing no relapse within 2 years after transplantation. The mean PASI scores of recurrent patients and non-recurrent patients after 2 years are shown by the lines labelled $\mathrm{b}$ and $\mathrm{c}$, respectively. PASI, psoriatic area and severity index.

\section{Adjustment of immunosuppressive regimens}

Non-recurrent cases. The three patients without PS recurrence were all diagnosed with HCC and treated with SRL plus or minus MMF therapy. The initial treatment regimen was FK506+MMF+steroid for 1 month (the steroid was stopped within a week), subsequently switched to SRL with the FK506 decreased gradually and withdrawn at 6 months postoperation. The concentration of SRL did not go beyond $10 \mathrm{ng} / \mathrm{ml}$, in accordance with liver and renal functions, as well as the presence or absence of rejection and drug toxicity reactions. PS was controlled, and the scope of skin lesions reduced gradually within 6 months after surgery. The patients were fully stable and cured within 12 months, with no rejection or disease relapse (PS or HCC) (Fig. 4).

Recurrent cases. The recurrent cases were the two patients with hepatitis B and PCs. The initial treatment, employed for the first 6 months, was FK506 plus MMF, with the blood concentration of FK506 kept at $8-12 \mathrm{ng} / \mathrm{ml}$ and the dosage maintained at 2.0-3.5 mg per treatment, twice a day, which was in line with 


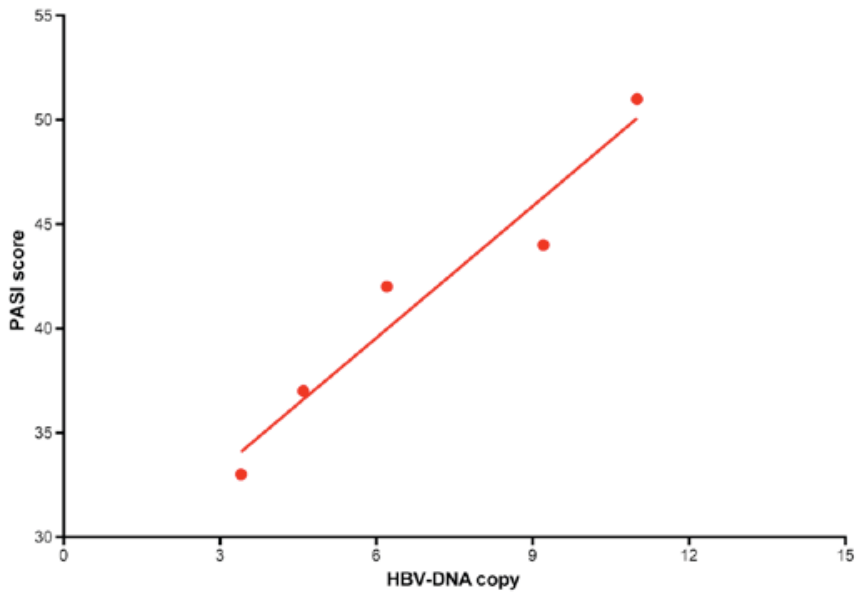

Figure 3. A scatter diagram of HBV DNA copy numbers vs. PASI scores. A positive correlation was found $(\mathrm{r}=0.97, \mathrm{P}=0.006)$. PASI, psoriatic area and severity index; HBV, hepatitis B virus.

the primary disease and the patients' weight. The graft survival and liver and renal functions remained stable at normal levels, while no obvious adverse drug reactions or rejections occurred. Furthermore, the PS was gradually controlled, and kept in remission and at stationary stages. During this period, the PS lesion areas became smaller, the color became weak, the inflammation reduced, and the scales gradually decreased. After the first 6 months, the immunosuppressive treatment was gradually switched to FK506 alone, with blood concentrations at 5.0-8.0 ng/ml within 12 months, and remaining at around $5.0 \mathrm{ng} / \mathrm{ml}$ after 12 months. This was gradually reduced, and stayed at 3.5-5.0 $\mathrm{ng} / \mathrm{ml}$ after 18 months, with regular detection of liver and kidney function. PS was well-controlled, with no progression.

These two patients underwent disease relapse with no obvious inducement at 22 and 24 months after LT. This may have been a result of the low FK506 concentration, so the dosage of FK506 was adjusted and maintained at almost $10 \mathrm{ng} / \mathrm{ml}$. However, the PS was not controlled well and became exacerbated. Another unexpected aspect was obvious nephrotoxicity and adverse drug reactions, but with no rejection. Therefore, based on our experience with HCC co-existing with PS, therapy was switched to SRL plus or minus MMF. Interestingly, the patients achieved remission and PS symptoms were gradually controlled after being treated for 3 months. The drug changes and monitoring index of one patient are shown in Fig 5.

\section{Discussion}

PS is a refractory dermatological disease with polygenic inheritance. It may be associated with virulence genes, environmental factors, infections, and immunologic derangement (1,3-5). The main pathological characteristics are excessive keratinocyte proliferation and parakeratosis, with recurring and repeated proliferation, desquamation and inflammation in the epidermis $(5,8,13)$. To the best of our knowledge, current treatments for PS are still limited in effect, which makes it difficult to control disease progression. Guidelines of care for the management of PS and psoriatic arthritis from the American

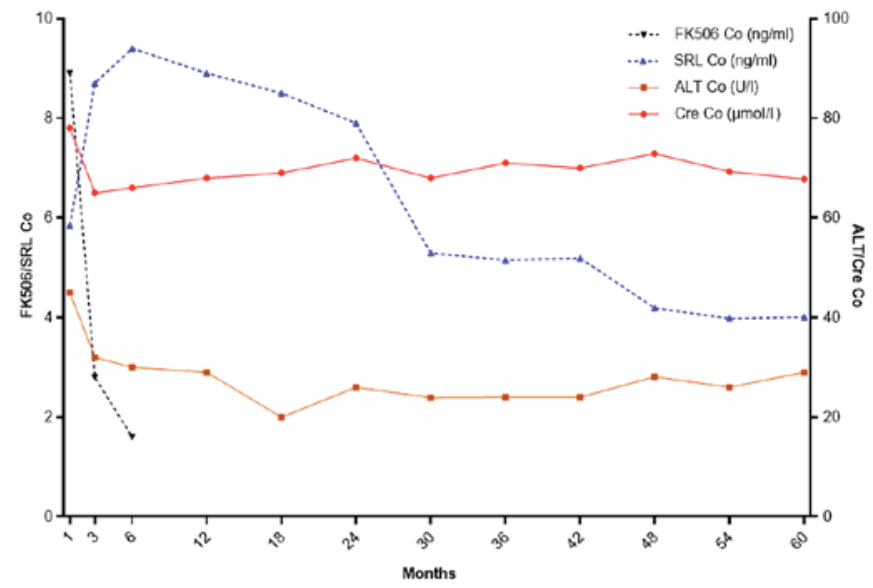

Figure 4. Changes in blood concentration, liver and kidney function of one liver transplantation patient with hepatocellular carcinoma (liver function index: ALT, U/1; kidney function index: Cre, $\mu \mathrm{mol} / 1)$. The FK506 and SRL concentrations (ng/ml) are displayed on the left, and the ALT and Cre concentrations on the right. ALT, alanine aminotransferase; FK506, tacrolimus; SRL, sirolimus; Co, concentration.

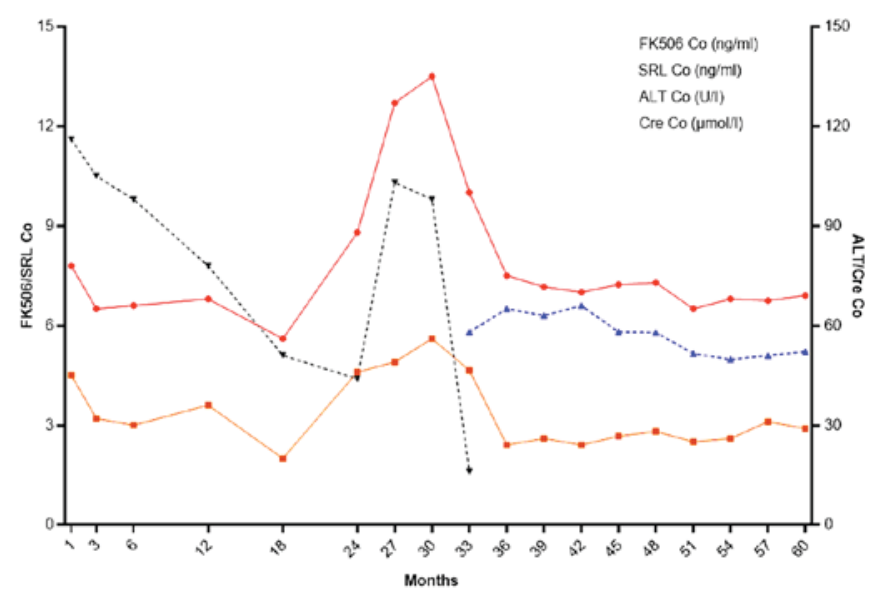

Figure 5. Changes in blood concentration, liver and kidney function of one liver transplantation patient with recurrent psoriasis (liver function index: ALT, U/1; kidney function index: Cre, $\mu \mathrm{mol} / 1)$. The FK506 and SRL concentrations $(\mathrm{ng} / \mathrm{ml})$ are displayed on the left, and the ALT and Cre concentrations on the right. ALT, alanine aminotransferase; FK506, tacrolimus; SRL, sirolimus; Co, concentration.

Academy of Dermatology (AAD) (2) suggest that the main treatment methods include topical medications, immunosuppression therapy (CsA and FK506), physical therapy and others, for example biotherapy. Traditional Chinese medicine is also used in China. The AAD Guidelines (2) also indicate that the purpose of therapy is to control the advance of PS, delay progression to whole body involvement, alleviate symptoms such as erythema, desquamation and local patch thickening, stabilize illness, avoid recurrence, avoid adverse effects, and improve patients' quality of life. Therefore, exploring effective treatments for PS has become the subject of intensive research and remains a difficult problem in immunology.

LT may cause patients to acquire a distinct and unique immune status, with a need for lengthy or lifelong immunosuppressive treatment, and also a requirement for the combination of at least two immunosuppressive agents (28). However, PS in the general population is rarely treated with more than one 
immunosuppressive agent, so LT patients with co-existing PS may undergo many more infections after the transplant operation. Another issue is that, if FK506 or CsA are used after LT, especially with long-term survival, the large dosages required for PS treatment often go beyond the maximum needed for the prevention of rejection, and may lead to drug toxicity reactions (infection and de novo cancer) and poor treatment effects. It has been reported that the immunosuppressive therapy for PS vulgaris mainly focuses on FK506 (29-32), but the evidence for co-existing PS after LT is relatively scarce (33-35). The limited literature describing FK506 treatment supplies some evidence, and shows that the long-term application of FK506 may lead to disease progression and repetition $(33,35)$. Therefore, combinational or single application immunosuppressive agents for these patients should be considered, taking into account their therapeutic effects, including PS control and rejection prevention, and their side effects.

Case reports from Hoover (22) and Collazo et al (21) indicate that etanercept can control post-transplantation PS progress effectively in patients with or without co-existing hepatitis $\mathrm{C}$ virus (HCV). The study by Foroncewicz et al (12) addressed the problem of PS recurrence when AID is associated with PS after LT, with a mean follow-up time of $7.80 \pm 1.48$ years. It indicated that, although long-term immunosuppressive therapy was applied after LT, there was still a possibility of PS recurrence. Foroncewicz et al (12) also suggested that the therapeutic effects of CsA are better than those of FK506 for patients with recurrence. Although, ten cases of recurrent PS were included to compare the therapeutic efficiency of CsA and tacrolimus after LT, but only two patients exhibited $\mathrm{HBV} / \mathrm{HCV}$ infection. So, the safety and efficacy of CsA still need to be further proved.

Research on the treatment of PCs or HCC co-existing with PS after LT is rarely reported. Therefore, the emphasis of our study lay on supplying different drug selections for different patients and reducing the toxic and side-effects of combinational drug applications. Two of five PS patients were preoperatively diagnosed with PCs and chronic hepatitis B. These patients were treated with FK506 plus a small dosage of MMF, for an extended period of time. When the FK506 dosage was reduced to prolong survival time, there was a recurrence of PS. In contrast, when the FK506 dosage was increased, PS was controlled but the nephrotoxicity of FK506 increased severely day by day. Instead of increasing FK506, switching to SRL for the relapsing patients controlled PS effectively. Three of five PS patients were preoperatively diagnosed with PCs and HCC. We used an SRL-based immunosuppressive treatment protocol for the purposes of prevention, treatment of postoperative tumor recurrence, and avoiding adverse drug reactions from lengthy application of FK506. The initial FK506+MMF treatment was switched to SRL 1 month after LT, and the MMF was reduced or withdrawn in accordance with patient and graft survival. The three patients underwent remission within 6 months, with a mean period $2.83 \pm 0.76$ months. During follow-up within $8.3 \pm 1.5$ years, no PS recurrence or progress occurred; in addition, no rejection and nephrotoxicity events emerged. Moreover, the SRL dosage was gradually stabilized by 2 years after LT. Overall, our analysis of the treatment of PCs and HCC co-existing with PS suggested that switching to SRL therapy not only prevented tumor relapse, but also effectively controlled PS pathogenesis. Moreover, it has been shown that dysregulation of the mechanistic target of rapamycin (mTOR) pathway plays a role in PS pathogenesis. mTOR is significantly increased in lesional skin and non-lesional skin, both at the gene and protein level $(26,27)$, and these alterations can be modulated by anti-TNF- $\alpha$ treatment (27). Therefore, we suggest that the SRL therapy could be used for ordinary PS patients as well as LT patients with PS, although its safety and efficacy for ordinary PS patients still remains to be proven.

Unlike FK506, the therapeutic concentration of SRL did not rely on drug dosage, but mainly on liver and renal function, as well as rejection status. Therefore, we suggest that SRL levels are maintained at or below $10 \mathrm{ng} / \mathrm{ml}$ to avoid interstitial pneumonia, thrombocytopenia and other severe adverse reactions. For the two patients with co-existing PCs and PS, because there was no evidence of HCC, we used the recommended immunosuppressive treatment of FK506 plus or minus MMF. As the immune tolerance of recipients to grafts following FK506 treatment displays an obvious time-dependence, the dosage was gradually decreased as time progressed. This may be the main reason that these two patients underwent postoperative PS relapse.

We discovered that, despite the fact that an FK506 concentration lower than $5 \mathrm{ng} / \mathrm{ml}$ at 1 year postoperation can benefit patients' survival, this concentration cannot effectively meet the need for PS control and inhibition to effect a cure and maintain a stable state. Moreover, the long-term lowering of drug concentration can lead to PS progression and recurrence. Previous case reports have indicated that postoperative blood concentrations of FK506 maintained at more than $10 \mathrm{ng} / \mathrm{ml}$ for extended times contributed to PS therapy; however, the patients were prone to adverse reactions which they could not tolerate, leading to withdrawal or reduction, which may lead to further PS progression. Therefore, considering our experience with previous cases of HCC with or without PS after LT, we tried SRL therapy for patients who had accepted FK506 therapy after LT, but without obvious effect, even after adjusting the dosage when undergoing PS relapse. The results proved to be satisfactory, the PS of these two patients were controlled at the resting stage 3 months later, and eventually reached cure levels. All of the above evidence suggests that immunosuppressive treatment based on SRL had a good curative effect on patients with co-exiting PS after LT, whether or not there is preoperative HCC, and that SRL may be used to replace FK506 for invalid patients or those repeated recurrence with no more therapy effect of FK506. For the small samples, when application with SRL therapy, it needs to be under strict surveillance of the drug concentration and adverse events.

That viral infections play important roles in PS pathophysiology in the general population is well recognized $(12,15,16)$. It has been reported that human immunodeficiency virus, $\mathrm{HCV}$, and herpes simplex virus 1 infections may trigger or even induce PS onset $(15,16)$, and it has also been observed that a direct relationship exists between PS activity and cytomegalovirus activation. Nevertheless, there is little research on HBV involvement in PS pathogenesis. Previous literature indicates that $\mathrm{HBV}$ infection may induce or aggravate PS progress (23-25) and HBV can be detected in PS skin lesions $(24,25)$. The patients included in this study were 
all diagnosed preoperatively as HBV-positive with lengthy chronic hepatitis B histories. Furthermore, their preoperative HBV copy numbers were positively correlated with PS severity. Therefore, HBIG was regularly injected postoperatively for all cases, to prevent hepatitis B recurrence. Although there is no evidence-based proof demonstrating a causal relationship between HBV and PS, the majority of PS patients are HBV positive. Therefore, anti-hepatitis B therapy may contribute to PS treatment; however, much remains to be done to explore the functional mechanism HBV in the occurrence of PS.

As a refractory dermatosis, PS co-exists with AIDs, especially ALD and scleroderma, so large dosages of immunosuppressant are often required to maintain effective treatment. Some experts contend that CsA is superior to FK506. However, we suggest here that PS therapy should follow a personalized medicine principle because of the few researches with rather small samples. There may be a better choice to combine CsA for selected patients with AID. Selection of an immunosuppressant should be in accordance with the severity of PS and the types of comorbidities. Application of an SRL-based immunosuppressant treatment may be a promising and beneficial approach for PS patients, regardless of LT status.

In summary, this study shows that SRL therapy can provide a superior therapeutic effect compared with FK506 treatment, for selected patients with co-existing PS after LT. This study also demonstrated that SRL therapy, even though given at lower doses and concentrations, could maintain PS in a cured or stable state in LT patients. Therefore, we conclude that SRL therapy may provide a promising new therapeutic approach for PS that may be superior to tacrolimus treatment. When co-existing $\mathrm{HBV}$ is found preoperatively, regularly injection of HBIG should be used to prevent HBV from relapsing or aggravating PS. There remains a need for further clinical studies with large samples to confirm the safety and efficacy of SRL therapy for co-existing PS after LT. Moreover, any application of SRL therapy for ordinary PS patients should be under strict surveillance, as further study is also needed to test and verify its benefit for these patients.

\section{Acknowledgements}

This study was supported by the Natural Science Foundation of China (nos. 81502376 and 81270509).

\section{References}

1. Lowes MA, Suárez-Fariñas M and Krueger JG: Immunology of psoriasis. Annu Rev Immunol 32: 227-255, 2014.

2. Menter A, Korman NJ, Elmets CA, Feldman SR, Gelfand JM, Gordon KB, Gottlieb A, Koo JY, Lebwohl M, Lim HW, et al: Guidelines of care for the management of psoriasis and psoriatic arthritis. Section 3. Guidelines of care for the management and treatment of psoriasis with topical therapies. J Am Acad Dermatol 60: 643-659, 2009.

3. Schommer NN and Gallo RL: Structure and function of the human skin microbiome. Trends Microbiol 21: 660-668, 2013.

4. Diani M, Altomare G and Reali E: T cell responses in psoriasis and psoriatic arthritis. Autoimmun Rev 14: 286-292, 2015.

5. Madankumar R, Teperman LW and Stein JA: Use of etanercept for psoriasis in a liver transplant recipient. JAAD Case Rep 1: S36-S37, 2015.

6. Diani M, Altomare G and Reali E: T Helper Cell Subsets in Clinical Manifestations of Psoriasis. J Immunol Res 2016: 7692024, 2016.
7. Lowes MA, Russell CB, Martin DA, Towne JE and Krueger JG: The IL-23/T17 pathogenic axis in psoriasis is amplified by keratinocyte responses. Trends Immunol 34: 174-181, 2013.

8. Kolios AG, Yawalkar N, Anliker M, Boehncke WH, Borradori L, Conrad C, Gilliet M, Häusermann P, Itin P, Laffitte E, et al: Swiss S1 guidelines on the systemic treatment of psoriasis vulgaris. Dermatology 232: 385-406, 2016.

9. Quesada JR and Gutterman JU: Psoriasis and alpha-interferon. Lancet 1: 1466-1468, 1986.

10. Baker BS, Griffiths CE, Lambert S, Powles AV, Leonard JN, Valdimarsson $\mathrm{H}$ and Fry L: The effects of cyclosporine A on T lymphocyte and dendritic cell subpopulations in psoriasis. Transplant Proc 20 (3 Suppl 4): S72-S77, 1988.

11. Balato A, Schiattarella M, Di Caprio R, Lembo S, Mattii M, Balato $\mathrm{N}$ and Ayala F: Effects of adalimumab therapy in adult subjects with moderate-to-severe psoriasis on Th17 pathway. J Eur Acad Dermatol Venereol 28: 1016-1124, 2014.

12. Foroncewicz B, Mucha K, Lerut J, Majewski S, Krawczyk M and Pączek L: Cyclosporine is superior to tacrolimus in liver transplant recipients with recurrent psoriasis. Ann Transplant 19: 427-433, 2014.

13. Yang ZS, Lin NN, Li L and Li Y: The effect of TNF inhibitors on cardiovascular events in psoriasis and psoriatic arthritis: An updated meta-analysis. Clin Rev Allergy Immunol 51: 240-247. 2016.

14. Krueger GG and Duvic M: Epidemiology of psoriasis: Clinical issues. J Invest Dermatol 102: 14S-18S, 1994.

15. Asadullah K, Prösch S, Audring H, Büttnerova I, Volk HD, Sterry W and Döcke WD: A high prevalence of cytomegalovirus antigenaemia in patients with moderate to severe chronic plaque psoriasis: An association with systemic tumour necrosis factor alpha overexpression. Br J Dermatol 141: 94-102, 1999.

16. Mehraein Y, Lennerz C, Ehlhardt S, Zang KD and Madry H: Replicative multivirus infection with cytomegalovirus, herpes simplex virus 1, and parvovirus B19, and latent Epstein-Barr virus infection in the synovial tissue of a psoriatic arthritis patient. J Clin Virol 31: 25-31, 2004.

17. Quintin E, Scoazec JY, Marotte H and Miossec P: Rare incidence of methotrexate-specific lesions in liver biopsy of patients with arthritis and elevated liver enzymes. Arthritis Res Ther 12: R143, 2010.

18. Howel D, Fischbacher CM, Bhopal RS, Gray J, Metcalf JV and James OF: An exploratory population based case-control study of primary biliary cirrhosis. Hepatology 31: 1055-1060, 2000.

19. Prince MI, Ducker SI and James OF: Case-control studies of risk factors for primary biliary cirrhosis in two United Kingdom populations. Gut 59: 508-512, 2010.

20. Reich K, Burden AD, Eaton JN and Hawkins NS: Efficacy of biologics in the treatment of moderate to severe psoriasis: A network meta-analysis of randomized controlled trials. Br J Dermatol 166: 179-188, 2012.

21. Collazo MH, González JR and Torres EA: Etanercept therapy for psoriasis in a patient with concomitant hepatitis $\mathrm{C}$ and liver transplant. P R Health Sci J 27: 346-347, 2008.

22. Hoover WD: Etanercept therapy for severe plaque psoriasis in a patient who underwent a liver transplant. Cutis 80: 211-214, 2007.

23. Steglich RB, Meneghello LP, Carvalho AV, Cheinquer H, Muller FM, Reginatto F: The use of ustekinumab in a patient with severe psoriasis and positive HBV serology. An Bras Dermatol. 89:652654, 2014.

24. Cho YT, Chen CH, Chiu HY, Tsai TF: Use of anti-tumor necrosis factor- $\alpha$ therapy in hepatitis B virus carriers with psoriasis or psoriatic arthritis: a case series in Taiwan. Dermatol. 39:269-273, 2012.

25. Cassano N, Mastrandrea V, Principi M, Loconsole F, De Tullio N, Di Leo A, Vena GA: Anti-tumor necrosis factor treatment in occult hepatitis B virus infection: a retrospective analysis of 62 patients with psoriatic disease. J Biol Regul Homeost Agents. 25:285-289, 2011

26. Balato A, Caprio RD, Lembo S, Mattii M, Megna M, Schiattarella M, Tarantino G, Balato N, Ayala F, Monfrecola G: Mammalian Target of Rapamycin in Inflammatory Skin Conditions. European Journal of Inflammation. 12:341-350,2014.

27. Balato A, Lembo S, Ayala F, Balato N, Caiazzo G, Raimondo A, Di Caprio R, Monfrecola G: Mechanistic target of rapamycin complex 1 is involved in psoriasis and regulated by anti-TNF- $\alpha$ treatment. Exp Dermatol. 26(4):325-327,2017. 
28. Geissler EK, Schnitzbauer AA, Zülke C, Lamby PE, Proneth A, Duvoux C, Burra P, Jauch KW, Rentsch M, Ganten TM: Sirolimus use in liver transplant recipients with hepatocellular carcinoma: A randomized, multicenter, open-label phase 3 trial. Transplantation. 100:116-125, 2016.

29. Lythgoe M, Abraham S: Tacrolimus: an effective treatment in refractory psoriatic arthritis following biologic failure. Clin Exp Rheumatol. 34: S12-S13, 2016.

30. Wei KC, Lai PC: Combination of everolimus and tacrolimus: a potentially effective regimen for recalcitrant psoriasis. Dermatol Ther. 28:25-27, 2015.

31. Tirado-Sánchez A, Ponce-Olivera RM: Preliminary study of the efficacy and tolerability of combination therapy with calcipotriene ointment $0.005 \%$ and tacrolimus ointment $0.1 \%$ in the treatment of stable plaque psoriasis. Cutis. 90: 140-144, 2012.

32. Laino L, Di Carlo A: Palmoplantar pustular psoriasis: clinical and video thermographic evaluation before and after topical tacrolimus treatment. Arch Dermatol. 147: 760,2011.
33. Foroncewicz B, Mucha K, Paczek L, Oldakowska-Jedynak U, Górnicka B, Zieniewicz K, Nyckowski P, Krawczyk M: AntiCD25 and tacrolimus therapy may not prevent early primary biliary cirrhosis recurrence after liver transplantation: two case reports. Transplant Proc. 35: 2310-2312, 2003.

34. Gilbert SC, Klintmalm G, Menter A, Silverman A: Methotrexateinduced cirrhosis requiring liver transplantation in three patients with psoriasis. a word of caution in light of the expanding use of this 'steroid-sparing' agent. Arch Intern Med. 150:889-891,1990.

35. Abu-Elmagd K, Van Thiel D, Jegasothy BV, Ackerman CD, Todo S, Fung JJ, Thomson AW, Starzl TE: FK506: a new therapeutic agent for severe recalcitrant psoriasis. Transplant Proc. 23:3322$3324,1991$. 